\title{
Research and application of the location information in the intelligent transportation
}

\author{
Junhan Wang \\ Tongji University \\ Shanghai, China \\ 326666501@qq.com
}

\author{
Fei Qiao, Jianfeng Lu \\ Tongji University \\ Shanghai, China \\ 1121wangjunhan@tongji.edu.cn
}

\begin{abstract}
- the traffic system is becoming quite complicated due to the increasing of roads and vehicles. Many countries realized Intelligent Transport Systems to make better use of existing transport resources. As Intelligent Transport Systems has mass traffic information data, it is a worthy study problem that how to make efficient use of these data. Because vehicles, the Individual of the Intelligent Transport System, have characteristics such as needing personalized information, mobile, huge number which make it difficult to publish information to those potential users. This paper uses Android tablet to be personal terminal of Intelligent Transport System. Using Android's GPS devices and various methods of wireless access such as GPRS, 3G, WIFI and so on, this personal terminal can connect to the Intelligent Transport Systems to search the latest real-time traffic information of the surrounding by using GPS information. What's more, those personal terminal also can sent traffic information to improve the Intelligent Transport Systems. Using the method of this paper can let individuals make efficient use of Intelligent Transport System, and ultimately achieve "safe travel".
\end{abstract}

Keywords- Android; Intelligent Transportation; GPS; Cloud Computing; Big Data

\section{INTRODUCTION}

Big city is building more and more roads and traffic infrastructures to deal with traffic congestion. For travelers, although a mass of roads and traffic infrastructures provide more choices for them, it also increases the complexity of the trip. Due to a lack of good information exchange and route guidance, invalid travel takes a large part of actual traffic behavior, which undoubtedly exacerbated the congestion and traffic accidents.

With the sustainable development of science and technology, we have some methods to solve this problem. We can get a lot of traffic information from Intelligent Transportation System to build up traffic information cloud platform. The development of mobile network makes connect to network in anytime, anywhere as possible. The emergence of tablet computers and smart phones makes the mobile personal network terminal has the very good implementation platform.

One of the most widely used tablet computers or smart phones OS these days is Android. Android is not only operating system but also middleware and key applications. Android, whose hardware is mainly based on ARM architecture platform, is a powerful Operating System supporting a large number of applications. Android has good UI in the user interaction design and system optimization. In the aspect of traffic information function, it can invoke Google MAP service which has precise GPS positioning system that can provide users with vehicle location. In terms of communication, Android support WIFI, Bluetooth, 3G and other communication methods, and support the TCP/IP protocol, URL to access network resources, as well as web service calls. According to the survey of Flurry, a Mobile application analysis company, Android device number has doubled in the past year, in April 2013, 564 million. Visible, As Android has lots of methods to connect to the Net, and can easy get GPS information, it is very accord with the requirement of this paper, and has great market potential. Therefore, based on the Android system to develop the personal vehicle application has very important practical significance.

People use radio to communicate with each other and receive real-time information, but radio can't send information targeted.

Nowadays, with the rapid development of mobile terminal software, more and more of the navigation software began to release real-time traffic conditions. However, although these software release information targeted, it is lack of interaction with the user. So people prefer to use radio.

Considering advantages and disadvantages of the above two methods, this paper uses mobile internet to connect to traffic information cloud platform, allows users real-time access to the network and allows users to update the traffic information, which guarantees the traffic information updated in real time, and improves the interaction between user and traffic information cloud platform. And using GPS information to search in the traffic information platform enable the software can be targeted.

\section{INSTRUCTION OF TRANSPORTATION INFORMATION SYSTEM}

This system consists of two parts: The Mobile Personal Terminal and Transportation Information Cloud Platform.

The Mobile Personal Terminal - This terminal is the entrance for individual users to connect to transportation information platform.

What the terminal do:

1 Upload the traffic information released by individual user. 
2 Use GPS information to search the surrounding traffic information.

Transportation Information Cloud Platform --- This terminal is used to store massive amounts of traffic information.

What the terminal do:

1 Receive the transmitted traffic information from personal mobile terminal.

2 Handle the request from mobile terminal and sent the search result of surrounding traffic information to vehicles.

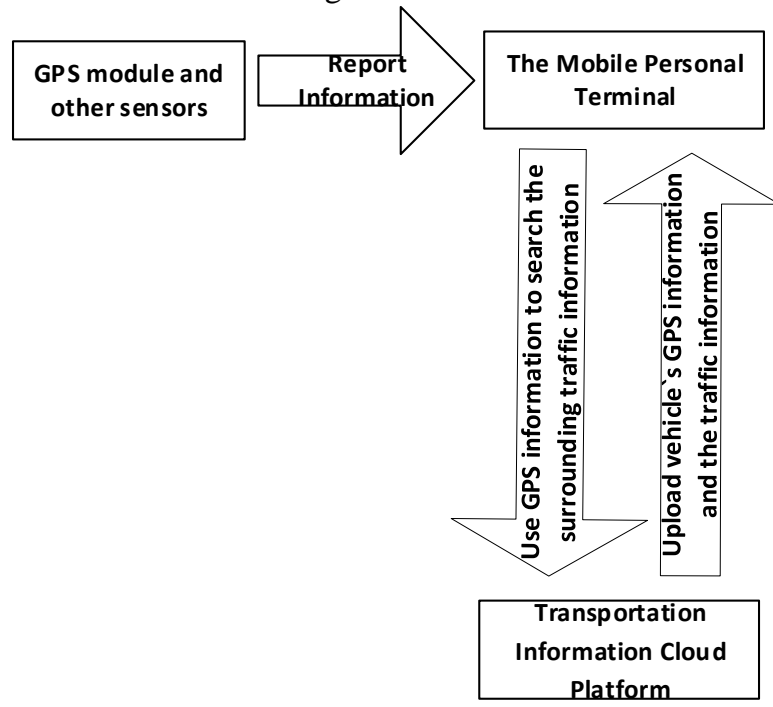

Figure 1. System's overall structure

\section{A. The design of The Mobile Personal Terminal}

In this paper, we use tablet equipped with android system to realize the Mobile Personal Terminal.

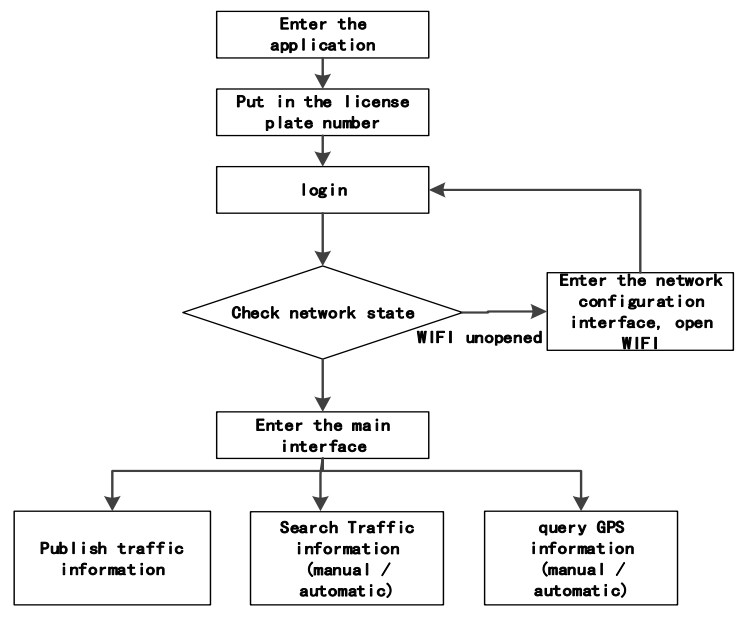

Figure 2. Software flow chart

As you can see from the diagram above, GPS module, Network test module and Communication module are the most important parts of this software.

\section{1) GPS module}

To use Android platform`s GPS device, we need to add the permissions, and then instantiate LocationManager, set parameters and use locationManager's requestLocationUpdates method to set automatic refresh listener. At all, we can use locationManager's getLastKnownLocation method to get the latitude and longitude information.

\section{2) Network test module}

Because the car has characteristics like fast speed, position uncertainly, it leads to network not stable. The software must be able to test the network's state. Considering some tablets without $3 \mathrm{G}$ function, the software assumes that network is provided by mobile $3 \mathrm{G}$ router. There are three parts of test.

First, the software needs to know whether the WIFI function is open. If it is not opening, the software will let user open WIFI. Only under the condition of WIFI opening, user can enter the main interface of the software.

Test code is as follows:

WifiManager wifimanager $=$

(WifiManager)context.getSystemService(Service.WIFI_ SERVICE);

int wifiFlag = wifimanager.getWifiState();

If wifiFlag`s value is 1 , that means WIFI is not open .

Second, the software need to Monitor network State, the results of ConnectivityManager's getAllNetworkInfo () method can be contrasted with the NetworkInfo. State. CONNECTED, if they are the same, the network connection is normal, otherwise software reminds users that the network connection is broken.

Third, the software uses try-catch block in data upload, capturing network error, and reminds users connect to the server is failed.

3) Communication module

As the network is not stable or may disconnect. Communication module also need to do something to improve user's experience. The Threads are used to let user to do other operations when the software is updating or searching.

Threads are the cornerstone of any multitasking operating system and can be thought of as mini-processes running within a main process, the purpose of which is to enable at least the appearance of parallel execution paths within applications.

In this situation, the code executing in a thread needs to interact with the user interface, it must do so by synchronizing with the main UI thread. This is achieved by creating a handler within the main thread, which, in turn, receives messages from another thread and updates the user interface accordingly.

The communication module made by information reporting module and surround searching module.

\section{a) information reporting module}

When user reports traffic information, the system starts up a thread to report information, the thread uses post method to submit a form to the traffic information cloud platform. After resolving the information, platform puts it 
into database, and automatically gives it not repeat number MSG_ID, which is used to determine the uniqueness of message. Then, the platform informs the client that uploading is success. In the end, the thread uses a handler to alert user the success of upload.

The form coded by com. Google. Gson. Gson package provides the toJson method to put object into JSON encoding, the format is as follows:

["MSG_ID ": "xxx ", "MSG_From ": "xxx ", "MSG_time ": "xxx", "Latitude1": "xxx ", "Longitude1": "xxx ",

"Latitude2": "xxx ", "Longitude2":"Xxx ", "MSG ": "XXX "].

As people always use meter to describe range, the software needs to put meter into latitude and longitude. 1 degrees in latitude is approximately equal to $111 \mathrm{~km}$, we can calculate to obtain the longitude and latitude of the form

4) surround searching module

This module is realized by a thread which starts after entering the software's main interface. Every 15 seconds, the thread submits a form to query surrounding information. The form contains the latitude and longitude of the user. Only when user's latitude and longitude in the message's area, the transportation information cloud platform returns the message in JSON format to the client. Client uses org. JSON. JSONObject's getString method to decode the information and store in the local database. Due to the local storage ability is limited and traffic information has timeliness, the software only keeps the latest 15 data. Users also can start the search manual by click the refresh button.

Search code is as follows:

Select $*$ from massage where Latitude $<$ Latitude 2 and $\mathrm{x}>$ Latitude 1 and Longitude < Longitude 2 and Longitude > Longitude 1

\section{B. The design of the Transportation Cloud Information Platform}

According to this scenario, the transportation information cloud platform needs a database to store transportation information. We use MySQL to build the database. And the core table is message table, which is used to store the traffic information updated by administrator and user.

The columns of this table are MSG_ID, MSG_From, MSG_time, Latitude1, Longitude1, Latitude2, Longitude2, MSG. MSG_ID used to determine the uniqueness of messages, MSG_From correspond two types of information, if the MSG comes from the administrator, the value of this column is "administrator". Or if the MSG comes from individual user, the value of this column is user's plate number. MSG_time is used to display when the MSG is edited. Latitude1 and Longitude1 are point 1's Latitude and Longitude, Latitude2 and Longitude2 are point 2`s Latitude and Longitude. Point 1 and point 2 are two diagonal vertices of a square, only in the square area, vehicles can receive this message.

\section{TEST OF THE TRANSPORTATION INFORMATION SYSTEM}

The system test environment is built by two Android tablets and one computer. Two Android tablet PC install the client software. computer is used to be a transportation information cloud platform.

Test steps are as follows:

(1) Two Android tablets respectively put into the user A's car and user B's car. After opened WIFI connection, user A enters the client's main interface by entering the vehicle license plate number. User B closes WIFI connection. Then he opens the software and inputs the vehicle license plate number, after the software's network test, he can't enter the main interface. After opened WIFI connection, user B enters the client's main interface.

(2) The platform administrator put three messages into the database. By limiting the area, we can simulate the three conditions of GPS intelligent searching surrounding information. The conditions are as fallow:

1 both $\mathrm{A}$ and $\mathrm{B}$ can receive the information.

2 both $\mathrm{A}$ and $\mathrm{B}$ can't receive the information.

$3 \mathrm{~B}$ can receive the information. But A can't.

(3) Waiting the search uses GPS information to search surrounding information, then $\mathrm{A}$ and $\mathrm{B}$ can receive the information from the platform administrator.

(4) A uses information reporting module to report the traffic information, and adjusts the range to ensure that $\mathrm{B}$ within the scope.

(5) waiting search uses GPS information to search surrounding information, B can see A`s traffic information (as shown in the figure below).

(6) The platform administrator puts 15 pieces of information into the database again, and set up the range, to ensure that both $\mathrm{A}$ and $\mathrm{B}$ can receive all of the information.

(7) Waiting search uses GPS information to search surrounding information, $\mathrm{A}$ and $\mathrm{B}$ receive the messages, and all old information is cleared.

(8) disconnected A`s WIFI connection, waiting the network test module works, the software prompts the user network connection is broken. After recovered WIFI connection, program prompts the user network connection is normal.

After the test, this system can test the network status, and realize the transportation information sent and selective receive.

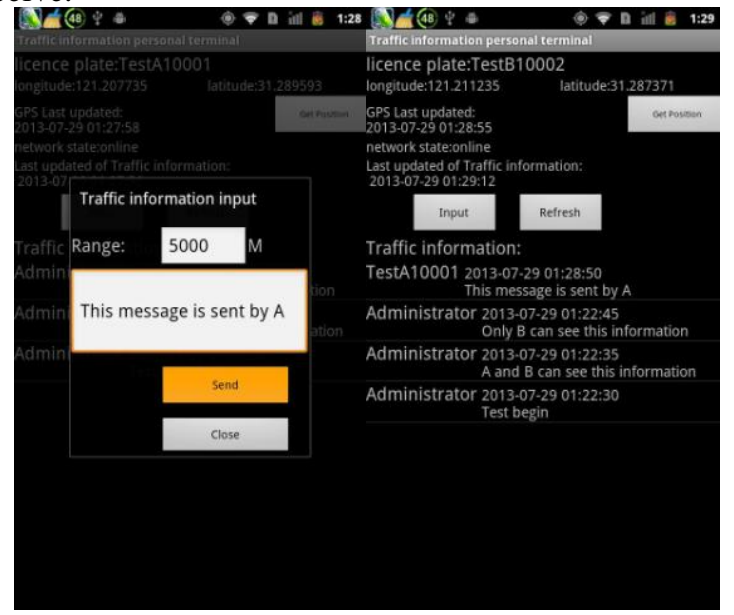

Figure 3. Test 


\section{CONCLUSION}

This paper designed a traffic information system which is based on Android platform and can interact with the net platform to send and receive information. This software also can detect the net's status. In this paper, because the software use the transportation information cloud platform which can be updated timely by the user and use the GPS information to do search, this software synthesizes the advantages of radio and navigation software by providing interactive platform, updating information timely and only sending the information to the surround users. As Android system has good portability and can be embedded in mobile phones and tablets, this study is valuable.

\section{ACKNOWLEDGMENT}

Collaborative multi-terminal network control platform and key technology under ubiquitous Network, Special issue of major national science and technology (No. 2011ZX03005-004-01)

\section{REFERENCES}

[1] Lauren Darcey Shane Conder ANDROID Wireless Application Development Pearson Education inc ISBN 978-0-321-74301-5

[2] IEEE 802.11p. WAVE-Wireless access for the vehicular environment [S]. 2008

[3] Xianhua,S,Zhenjun,D.,Rong,C.Research on mobile location service design based on Android. Proceedings - 5thInt. Conf. WiCOM 2009. 2009

[4] IEEE Android Based Wireless Location and Surrounding Search System Design 2010

[5] Code Home Android-An Open Handset Alliance Project. http://code.google.com/android/what-is-an-droid.html . 2008

[6] Android 4.2 App Development Essentials 2013 ISBN 1491075376

[7] Google Android [M]. E2ECloud Studio, BeiJing: Posts\&Telecom Press.2009

[8] Li Xudong,Yan Gaoshi,Tang Hai.Android Based Wireless Location and SurroundingSearch System Design. Procedings of the Ninth International Symposium onDistributed Computing and Applications to Business, Engineering and Science . 2010

[9] Nong Li-ping, Wang Li-hu, Huang Y-iping, "Application re-search of Android in embedded vehicle navigation system"[D] BeiJing: Computer Engineering\&Design, 2010, 31(11).

[10] SunWei-qin, Tomcat\&Java Web development of technologysolutions [M]. BeiJing: Publishing House of Electronics Indus-try. 